\title{
ORBITALLY NONEXPANSIVE MAPPINGS
}

\author{
ENRIQUE LLORENS-FUSTER
}

(Received 29 July 2015; accepted 27 August 2015; first published online 11 November 2015)

\begin{abstract}
We define a class of nonlinear mappings which is properly larger than the class of nonexpansive mappings. We also give a fixed point theorem for this new class of mappings.
\end{abstract}

2010 Mathematics subject classification: primary 47H09; secondary 37C25, 47H10.

Keywords and phrases: fixed point, nonexpansive mapping, normal structure.

\section{Introduction}

Nonexpansive mappings are those which have Lipschitz constant equal to one. For example, contractions, isometries and the resolvents of accretive operators are all nonexpansive. In the setting of Banach spaces, the fixed point theory for nonexpansive mappings has been an object of extensive research because, amongst other reasons, there is a deep connection between these fixed point results and the geometric properties of the norm of the Banach space where the mappings are defined.

Several generalisations of nonexpansive mappings have received attention, for example those due to Goebel and Kirk [7], Goebel et al. [8], Suzuki [11], GarcíaFalset et al. [6] and Aoyama and Kohsaka [1]. The goal of these generalisations is simply to enlarge the class of mappings for which the fixed point results (existence and convergence of some iteration process) hold. In 2011, Llorens-Fuster and MorenoGálvez [10] defined the so-called $L$-type mappings. This is a quite general class of mappings which properly contains most of the previously defined families of generalised nonexpansive mappings.

To check that a given mapping belongs to any of these classes of generalised nonexpansive mappings can often be a hard task. Here we will define a different class of (nonlinear) mappings for which this task seems to be easier. We have called them orbitally nonexpansive and they properly contain the nonexpansive mappings and also the $L$-type mappings in some cases.

The author has been supported by the grant MTM2012-34847-C02-02.

This is an Open Access article, distributed under the terms of the Creative Commons Attribution licence (http://creativecommons.org/licenses/by/4.0/), which permits unrestricted re-use, distribution, and reproduction in any medium, provided the original work is properly cited.

(C) 2015 Australian Mathematical Publishing Association Inc. 0004-9727/2015 \$16.00 
We will give a fixed point result for orbitally nonexpansive mappings in reflexive Banach spaces and we will also give an example of a fixed point free orbitally nonexpansive selfmapping of a closed, convex and bounded subset of a reflexive Banach space. This is a remarkable feature of this class of mappings, because it is unknown if there exists a nonexpansive fixed point free mapping under these conditions.

\section{Preliminaries}

Throughout this paper, we suppose that $(X,\|\cdot\|)$ is a real Banach space and $0_{X}$ its zero vector. If $A$ is a nonempty subset of $X, \operatorname{conv}(A)$ will denote the convex hull of the set $A$. From now on, $C$ stands for a given nonempty, closed, convex and bounded subset of $X$. A mapping $T: C \rightarrow X$ is nonexpansive if for all $x, y \in C,\|T(x)-T(y)\| \leq\|x-y\|$. If $T: C \rightarrow C$ is a mapping and $x_{0} \in C$, the sequence $\left(T^{n}\left(x_{0}\right)\right)$ is often called the orbit of $T$ starting at $x_{0}$. If $T: C \rightarrow X$ is a mapping, a sequence $\left(x_{n}\right)$ in $C$ is called an almost fixed point sequence (a.f.p.s. for short) for $T$ in $C$ whenever $x_{n}-T\left(x_{n}\right) \rightarrow 0_{X}$. It is well known that if $T: C \rightarrow C$ is nonexpansive and $D \subset C$ is nonempty, closed, convex and $T$-invariant (that is, $T(D) \subset D$ ), then $T$ has almost fixed point sequences in $D$.

Definition 2.1 [10]. We say that the mapping $T: C \rightarrow C$ is an $L$-type mapping (or that $T$ satisfies condition $(L))$ on $C$ provided that:

(a) if $D \subset C$ is nonempty, closed, convex and $T$-invariant, then $T$ has an a.f.p.s. $\left(x_{n}\right)$ in $D$;

(b) for every a.f.p.s. $\left(x_{n}\right)$ for $T$ in $C$ and, for each $x \in C$,

$$
\limsup _{n \rightarrow \infty}\left\|x_{n}-T(x)\right\| \leq \limsup _{n \rightarrow \infty}\left\|x_{n}-x\right\| .
$$

The hypothesis (a) was called Condition (A) by Dhompongsa and Nanan in [4]. To check that a given mapping satisfies this condition can often become a hard task. However, Condition (A) is automatically satisfied by several classes of mappings. For instance, if a mapping $T: C \rightarrow C$ is nonexpansive with respect to some equivalent renorming of $X$, then $T$ satisfies Condition (A).

A mapping $T: C \rightarrow C$ is said to be asymptotically regular provided that for every $x \in C, T^{n+1}(x)-T^{n}(x) \rightarrow 0_{X}$. Of course, asymptotically regular mappings satisfy Condition (A). Moreover, $T$ is said to be quasi-nonexpansive provided that $T$ has at least one fixed point in $C$ and, if $p \in C$ is such a fixed point, then, for all $x \in C$, $\|p-T(x)\| \leq\|p-x\|$. This concept was essentially introduced (along with some related ideas) by Díaz and Metcalf [5] in 1967.

To finish this section, we recall a relevant geometric property of Banach spaces. A Banach space $(X,\|\cdot\|)$ is said to have normal structure if each nonempty, bounded, closed and convex subset $C$ of $X$ with $\operatorname{diam}(C)>0$ contains a point $p \in C$ such that $r_{C}(p):=\sup \{\|p-x\|: x \in C\}<\operatorname{diam}(C)$. Every uniformly convex Banach space has normal structure, but the converse is not true. 
This property is relevant in metric fixed point theory because of the seminal work of Kirk [9], who showed that every nonexpansive selfmapping of a nonempty weakly compact convex set of a Banach space with normal structure has a fixed point. Similar results for several kinds of generalised nonexpansive mappings were obtained in [3] and [10].

\section{Orbitally nonexpansive mappings}

Several definitions of generalised nonexpansive mappings are concerned with the iterates of the mapping under consideration and hence they are related to the behaviour of its orbits. For instance, a mapping $T: C \rightarrow C$ is said to be asymptotically nonexpansive (see [7]) if for all $x, y \in C,\left\|T^{n}(x)-T^{n}(y)\right\| \leq k_{n}\|x-y\|$, where $\left(k_{n}\right)$ is a sequence of real numbers such that $\lim k_{n}=1$.

Here we will present a class of mappings for which some of its orbits behave as a kind of attractor.

Definition 3.1. A mapping $T: C \rightarrow C$ is said to be orbitally nonexpansive if for every nonempty, closed, convex, $T$-invariant subset $D$ of $C$, there exists some $x_{0} \in D$ such that for every $x \in D$,

$$
\limsup _{n \rightarrow \infty}\left\|T^{n}\left(x_{0}\right)-T(x)\right\| \leq \limsup _{n \rightarrow \infty}\left\|T^{n}\left(x_{0}\right)-x\right\| .
$$

The following results are obvious.

Proposition 3.2. If $T: C \rightarrow C$ is nonexpansive, then $T$ is orbitally nonexpansive. In particular, every contraction and every constant mapping is orbitally nonexpansive.

Proposition 3.3. If $T: C \rightarrow C$ is an asymptotically regular L-type mapping, then $T$ is orbitally nonexpansive.

Proof. If $D$ is a nonempty, closed, convex, $T$-invariant subset of $C$, we may choose any $x_{0} \in D$ and then $\left(T^{n}\left(x_{0}\right)\right)$ is an a.f.p.s. because $T$ is asymptotically regular. Moreover, by condition $(L)$, for every $x \in D$,

$$
\underset{n}{\lim \sup }\left\|T^{n}\left(x_{0}\right)-T(x)\right\| \leq \underset{n}{\lim \sup }\left\|T^{n}\left(x_{0}\right)-x\right\|,
$$

which means that $T$ is orbitally nonexpansive.

There are continuous orbitally nonexpansive mappings which are not Lipchitzian.

Example 3.4. Let $T:[0,1] \rightarrow[0,1]$ be given by $T(x)=\sqrt{x}$. The closed convex $T$ invariant subsets of $[0,1]$ are just all the intervals of the form $[a, 1]$ with $a \in[0,1]$. Choosing $x_{0}=1$ gives $T^{n}\left(x_{0}\right) \equiv 1$. Therefore, for every $x \in[a, 1]$,

$$
\limsup _{n \rightarrow \infty}\left|T^{n}\left(x_{0}\right)-T(x)\right|=|1-\sqrt{x}|=\frac{1-x}{1+\sqrt{x}} \leq|1-x|=\limsup _{n \rightarrow \infty}\left|T^{n}\left(x_{0}\right)-x\right|
$$

and $T$ is an orbitally nonexpansive mapping. On the other hand, $0 \in \operatorname{Fix}(T)$ and, for every $x \in(0,1),|0-\sqrt{x}|>|0-x|$. Thus, the mapping $T$ is not quasi-nonexpansive and 
hence fails to satisfy condition $(L)$. Moreover, the mapping $T$ is asymptotically regular. Indeed, for every $x \in(0,1)$,

$$
\lim _{n}\left|T^{n+1}(x)-T^{n}(x)\right|=\lim _{n}\left|x^{2^{-n-1}}-x^{2^{-n}}\right|=\lim _{n} x^{2^{-n-1}}\left|1-x^{2^{-n-1}}\right|=0
$$

and the same equality holds for the trivial cases $x=0$ and $x=1$. Thus, the asymptotic regularity alone is not sufficient for condition $(L)$. From this example it follows that the assumption of condition $(L)$ is not superfluous in Proposition (3.3).

Proposition 3.5. Let $(X,\|\cdot\|)$ be a Banach space with normal structure. Let $C$ be a nonempty, weakly compact, convex subset of $X$. Then every L-type mapping $T: C \rightarrow C$ is orbitally nonexpansive.

Proof. Let $D$ be a nonempty, closed, convex $T$-invariant subset of $C$. Since $T$ is an $L$ type mapping, from [10, Theorem 4.4], $T$ has a fixed point $x_{0} \in D$. Then $T^{n}\left(x_{0}\right) \equiv x_{0}$ and therefore, for every $x \in D$, bearing in mind that $L$-type mappings are quasinonexpansive,

$$
\underset{n}{\lim \sup }\left\|T^{n}\left(x_{0}\right)-T(x)\right\|=\left\|x_{0}-T(x)\right\| \leq\left\|x_{0}-x\right\|=\underset{n}{\lim \sup }\left\|T^{n}\left(x_{0}\right)-x\right\|,
$$

which implies that $T$ is orbitally nonexpansive.

Notice that orbitally nonexpansive mappings need not be continuous, as [11, Example 1] and [10, Proposition 3.4], along with the above proposition, show.

\section{Fixed point results}

First of all, let us point out that there are orbitally nonexpansive fixed point free mappings. Indeed, in Example 4.3 below, we will give an example which leaves invariant a weakly compact convex subset of a reflexive Banach space.

THEOREM 4.1. Let $K$ be a nonempty weakly compact convex subset of a Banach space $(X,\|\cdot\|)$ with normal structure. Let $T: K \rightarrow K$ be an orbitally nonexpansive mapping. Then $T$ has a fixed point.

Proof. Since $K$ is a weakly compact set, from a standard application of Zorn's lemma, there is a nonempty, closed, convex, $T$-invariant subset $C$ of $K$ with no proper subsets enjoying these characteristics. From the definition of orbitally nonexpansive mapping, there exists $x_{0} \in C$ such that, for every $x \in C$,

$$
\limsup _{n \rightarrow \infty}\left\|T^{n}\left(x_{0}\right)-T(x)\right\| \leq \limsup _{n \rightarrow \infty}\left\|T^{n}\left(x_{0}\right)-x\right\| .
$$

We will distinguish two cases.

Case I. There exists $z \in C$ such that $T^{n}\left(x_{0}\right)=z$ for $n$ large enough. We claim that, in this case, $z$ is a fixed point of $T$. Indeed,

$$
\|z-T(z)\|=\limsup _{n \rightarrow \infty}\left\|T^{n}\left(x_{0}\right)-T(z)\right\| \leq \limsup _{n \rightarrow \infty}\left\|T^{n}\left(x_{0}\right)-z\right\|=0 .
$$


Case II. The sequence $\left(T^{n}\left(x_{0}\right)\right)$ is bounded and not (eventually) constant. Since the Banach space $(X,\|\cdot\|)$ has normal structure, from [3, Corollary 1], the real function $g: C \rightarrow[0, \infty)$ defined by

$$
g(x):=\limsup _{n \rightarrow \infty}\left\|x-T^{n}\left(x_{0}\right)\right\|
$$

is not constant on $\operatorname{conv}\left\{T^{n}\left(x_{0}\right): n=1,2, \ldots\right\}$. Then $g$ takes at least two different real values, that is, there exist $v_{1}, v_{2} \in \operatorname{conv}\left\{T^{n}\left(x_{0}\right): n=1,2, \ldots\right\} \subset C$ such that

$$
r_{1}:=g\left(v_{1}\right)<g\left(v_{2}\right)=: r_{2} \text {. }
$$

Let $r:=\frac{1}{2}\left(r_{1}+r_{2}\right)$ and consider the set

$$
M:=\{x \in C: g(x) \leq r\} .
$$

It is straightforward to check that $M$ is nonempty, closed and convex and $M \neq C$ because $v_{2} \notin M$. Moreover, for every $x \in M$, since $T$ is an orbitally nonexpansive mapping,

$$
g(T(x))=\limsup _{n \rightarrow \infty}\left\|T(x)-T^{n}\left(x_{0}\right)\right\| \leq \limsup _{n \rightarrow \infty}\left\|x-T^{n}\left(x_{0}\right)\right\|=g(x) \leq r .
$$

Thus, $M$ is a nonempty, closed, convex and $T$-invariant subset of $C$ with $M \neq C$, which is a contradiction to the minimality of $C$. Thus, Case II is not possible.

We finish with two examples. The first is an orbitally nonexpansive mapping in a Hilbert space (and hence for which Theorem 4.1 applies because every Hilbert space has normal structure), but it does not fall into the scope of most of the standard fixed point theorems.

EXAMPLE 4.2. In the standard Hilbert space $\ell_{2}$, consider the set

$$
C=\left\{x=\left(x_{n}\right) \in \ell_{2}:\|x\|_{2} \leq 1, x_{n} \geq 0, n=1,2, \ldots\right\},
$$

where $\|x\|_{2}=\left(\sum_{n=1}^{\infty}\left|x_{n}\right|^{2}\right)^{1 / 2}$ is the ordinary Euclidean norm of $x=\left(x_{n}\right) \in \ell_{2}$. Let $T: C \rightarrow C$ be the mapping defined by $T\left(\left(x_{n}\right)\right)=\left(x_{n}^{2}\right)$.

Notice that the origin $0_{\ell_{2}}$ along with the elements of the standard Schauder basis $\left\{e_{n}\right\}$ are just the fixed points of $T$. It is easy to check that $T$ fails to be quasi-nonexpansive and, according to [10, page 5], $T$ cannot be an $L$-type mapping.

It is obvious that, for every $\left(x_{n}\right) \in C$,

$$
\left\|T\left(\left(x_{n}\right)\right)\right\|^{2}=\sum_{n=1}^{\infty} x_{n}^{4} \leq \sum_{n=1}^{\infty} x_{n}^{2}=\left\|\left(x_{n}\right)\right\|^{2} .
$$

Let $D$ be a nonempty, closed, convex, $T$-invariant subset of $C$. Then either $0_{\ell_{2}} \in D$ or $D=\left\{e_{k}\right\}$. In the first case, choosing $x_{0}=0_{\ell_{2}} \in D$, for all $y \in D$,

$$
\lim \sup \left\|T^{n}\left(x_{0}\right)-T(y)\right\|_{2}=\|T(y)\|_{2} \leq\|y\|_{2}=\lim \sup \left\|T^{n}\left(x_{0}\right)-y\right\|_{2} .
$$

In the second case, $D$ is a singleton and trivially (3.1) is satisfied. Thus, $T$ is an orbitally nonexpansive selfmapping of $C$ but it is not nonexpansive. 
The following example shows that the assumption of normal structure cannot be avoided in Theorem 4.1 .

ExAmple 4.3. Let $X_{2}$ be the ordinary Hilbert space $\ell_{2}$ endowed with the norm

$$
|x|:=\max \left\{\|x\|_{2}, 2\|x\|_{\infty}\right\}
$$

where, for $x=\left(x_{n}\right) \in \ell_{2},\|x\|_{\infty}=\sup \left\{\left|x_{n}\right|: n=1,2, \ldots\right\}$. It is obvious that $X_{2}$ is a Banach space which is isomorphic to $\left(\ell_{2},\|\cdot\|_{2}\right)$. It is well known that $X_{2}$ fails to have normal structure (see [2]).

For $x \in \ell_{2}$, define $S(x):=\left\{n \in \mathbb{Z}^{+}:\left|x_{n}\right|=\|x\|_{\infty}\right\}$. If $x \neq 0_{\ell_{2}}$, then $S(x)$ is a nonempty finite set and we can define $p(x):=\max S(x)$. In particular, $p\left(\frac{1}{2} e_{k}\right)=k$.

Let $K:=\left\{x \in \ell_{2}:|x| \leq 1, x_{n} \geq 0, n=1,2, \ldots\right\}$. The set $K$ is weakly compact and convex in $\ell_{2}$. Let us consider the mapping $T: K \rightarrow K$ defined by $T\left(0_{\ell_{2}}\right)=\frac{1}{2} e_{1}$ and, for $x \neq 0_{\ell_{2}}$,

$$
T(x)=\frac{1}{2} e_{p(x)+1} .
$$

It is obvious that $T$ maps $K$ into itself and that $T$ has no fixed points. In fact, for every $x \in K, x \neq 0_{\ell_{2}}$,

$$
T(x)=\frac{1}{2} e_{p(x)+1}, T^{2}(x)=\frac{1}{2} e_{p(x)+2}, \ldots, T^{k}(x)=\frac{1}{2} e_{p(x)+k} .
$$

For $x=0_{\ell_{2}}, T(x) \neq 0_{\ell_{2}}$ and therefore $T^{k+1}(x)=\frac{1}{2} e_{p(x)+k}$. Then, for $n$ large enough and $x, y \in K$,

$$
\left\|T^{n}(x)-y\right\|_{2}^{2}=\frac{1}{4}+\|y\|_{2}-2\left\langle T^{n}(x), y\right\rangle=\frac{1}{4}+\|y\|_{2}-y_{p(x)+n}
$$

and

$$
\left\|T^{n}(x)-T(y)\right\|_{2}^{2}=\frac{1}{2} .
$$

On the other hand, also for $n$ large enough, $2\left\|T^{n}(x)-T(y)\right\|_{\infty}=1$. Moreover,

$$
2\left\|T^{n}(x)-y\right\|_{\infty}=2\left\|\left(y_{1}, \ldots, y_{p(x)}, \ldots, \frac{1}{2}-y_{n+p(x)}, y_{n+p(x)+1}, \ldots\right)\right\|_{\infty} \rightarrow 1 .
$$

This implies that

$$
\limsup _{n \rightarrow \infty}\left|T^{n}(x)-T(y)\right|=\max \left\{\sqrt{\frac{1}{2}}, 1\right\}=1
$$

while

$$
\limsup _{n \rightarrow \infty}\left|T^{n}(x)-y\right|=\max \left\{\sqrt{\frac{1}{4}+\|x\|_{2}}, 1\right\} \geq 1
$$

and it follows immediately that $T$ is a fixed point free orbitally nonexpansive mapping on $K$ with respect to the norm $|\cdot|$. 


\section{Concluding remark}

To be an orbitally nonexpansive mapping is a metric property, that is, an orbitally nonexpansive mapping with respect to a given norm in a Banach space $X$ may lose this property after an equivalent renorming of $X$. For instance, the $|\cdot|$-orbitally nonexpansive mapping $T$ considered in the last example is fixed point free. According to Theorem 4.1, $T$ cannot be orbitally nonexpansive with respect to the Euclidean norm $\|\cdot\|_{2}$, which enjoys normal structure.

A Banach space is said to have the fixed point property for nonexpansive mappings (FPP in short) if, for every nonempty, closed, convex, bounded subset $C$ of $X$, every nonexpansive mapping $T: C \rightarrow C$ has a fixed point. In a similar way, the fixed point property with respect to orbitally nonexpansive mappings (ONE-FPP) could be defined. Notice that it is a famous open question whether every reflexive Banach space enjoys FPP. While the space $X_{2}$ of the last example enjoys FPP (see [2]), it is obvious that it fails ONE-FPP. This raises the question of characterising the Banach spaces enjoying this property.

\section{References}

[1] K. Aoyama and F. Kohsaka, 'Fixed point theorem for $\alpha$-nonexpansive mappings in Banach spaces', Nonlinear Anal. 74 (2011), 4387-4391.

[2] J. B. Baillon and R. Schoneberg, 'Asymptotic normal structure and fixed points of nonexpansive mappings', Proc. Amer. Math. Soc. 81 (1981), 257-264.

[3] J. Bogin, 'A generalization of a fixed point theorem of Goebel, Kirk and Shimi', Canad. Math. Bull. 19 (1976), 7-12.

[4] S. Dhompongsa and N. Nanan, 'Fixed point theorems by ways of ultra-asymptotic centers', Abstr. Appl. Anal. 2011 (2011), Article ID 826851, 21 pages, doi:10.1155/2011/826851.

[5] J. B. Díaz and F. T. Metcalf, 'On the structure of the set of subsequential limit points of successive approximations', Bull. Amer. Math. Soc. 73 (1967), 516-519.

[6] J. García-Falset, E. Llorens-Fuster and T. Suzuki, 'Fixed point theory for a class of generalized nonexpansive mappings', J. Math. Anal. Appl. 375 (2011), 185-195.

[7] K. Goebel and W. A. Kirk, 'A fixed point theorem for asymptotically nonexpansive mappings', Proc. Amer. Math. Soc. 35 (1972), 171-174.

[8] K. Goebel, W. A. Kirk and T. N. Shimi, 'A fixed point theorem in uniformly convex spaces', Boll. Unione Mat. Ital. (9) 7(4) (1973), 67-75.

[9] W. A. Kirk, 'A fixed point theorem for mappings which do not increase distance', Amer. Math. Monthly 72 (1965), 1004-1006.

[10] E. Llorens-Fuster and E. Moreno-Gálvez, 'The fixed point theory for some generalized nonexpansive mappings', Abstr. Appl. Anal. 2011 (2011), Article ID 435686, 15 pages, doi:10.1155/2011/435686.

[11] T. Suzuki, 'Fixed point theorems and convergence theorems for some generalized nonexpansive mappings', J. Math. Anal. Appl. 340 (2008), 1088-1095.

\section{ENRIQUE LLORENS-FUSTER, Department of Mathematical Analysis,}

University of Valencia, Dr. Moliner 50,

46100 Burjassot, Valencia, Spain

e-mail: Enrique.Llorens@uv.es 